$\xi$

\title{
A Systematic Review for Computer Based Observational Techniques for Assessing Exposure to Risk Factors for Work- Related Musculoskeletal Disorders
}

\author{
Syahrul Aziana Abdul Rahman and Mohd Nasrull Abdol Rahman \\ Department of Manufacturing and Industrial Engineering, Faculty \\ Of Mechanical and Manufacturing Engineering, Universiti Tun \\ Hussien Onn Malaysia (UTHM), 86400 Batu Pahat, Johor, Malaysia
}

\begin{abstract}
The objective of this review are to systematically examine the existing techniques of computer based observational method for assessing Work-related musculoskeletal disorders (WMSDs) and analysed them to the needs of different potential users. Articles related are searched and collected from scientific database starting from 1977 to 2016. Seven methods are identified for computer based observational techniques and from these methods, only three methods have been evaluated as the intra-observer reliability and five methods are evaluated as interobserver reliability where the average results are moderate to good agreement. For concurrent validity, five methods have been evaluated with moderate agreement. Some of the risk factors that related with WMSDs are; physical, psychosocial, work organization and individual factors. In addition, these existing techniques did not fulfil the criteria of reliability and validity testing during the development of these methods.
\end{abstract}

Keywords: Computer based observational methods, risk factors, work-related muskuloskeletal disorders (WMSDs), reliability, validity

\section{Introduction}

Work-related musculoskeletal disorders (WMSDs) closely linked to various environmental factors and working conditions that can cause the function of musculoskeletal system and there are many risk factors at work such as; physical [1-5], psychosocial [6, 7], work organizational $[6,8,9]$, and individual factor [3, 10-12] that contribute to the WMSDs $[3,8]$.

Various computer-based observation techniques have been established for the evaluation of exposure to risk factors of WMSDs during work [13-20]. These techniques have been widely used in workplaces, easy and fast to be applied [16, 19, 21-23]. Vedder has stated that these methods is a convenient, time saving tool and reduce errors within observation [24].

Therefore, the objective of this reviewed are to systematically examine the existing techniques of computer based observational method for assessing WMSDs and analysed them to the needs of different potential users.

\section{Methods}

\subsection{Search and Selection of Reference Literature}

For this research, the reference literature are searched from scientific database using the following search engines, such as; ScienceDirect, Scopus, Google, Google Scholar, Wiley Online Library and PubMed.
It is done by using several coalitions of keywords in Boolean operator methods that related to computer based observational methods for assessing exposure to risk factors of WMSDs. This method basically uses three main operators which are; AND, OR, and NOT [25]. AND operator allows the searching of documents containing two distinct words such as; computer-aided AND observational methods and computer-based AND evaluation tools. Secondly, the OR operator is used to find information that seems to be the same, for instance; evaluation tools OR assessment methods, and computer-based OR video-based. The studies were executed by comprising several computer based observational methods from year 1977 to 2016. Figure 1 are demonstrated the flow diagram for search and selection of reference literature. As a result, about 450 articles are found of database searching resulted, but only 114 potentials references were screened according to the title and abstract, while a total 73 articles were full text screening. The others 41 articles are not related between the exposures of risk factors with WMSDs. The inclusion criteria were followed based on the framework that has been developed by research in Section 2.2.

\subsection{Developing the Framework for Evaluation}

The framework of evaluation for the methods in this review has been developed by researchers. The basic items for structure and contents of this frameworks includes the exposure of risk factors related to WMSDs which are; physical, psychosocial, individual and work organization factors, the validity and reliability testing, and the 
strength and limitation of the computer based observational method.

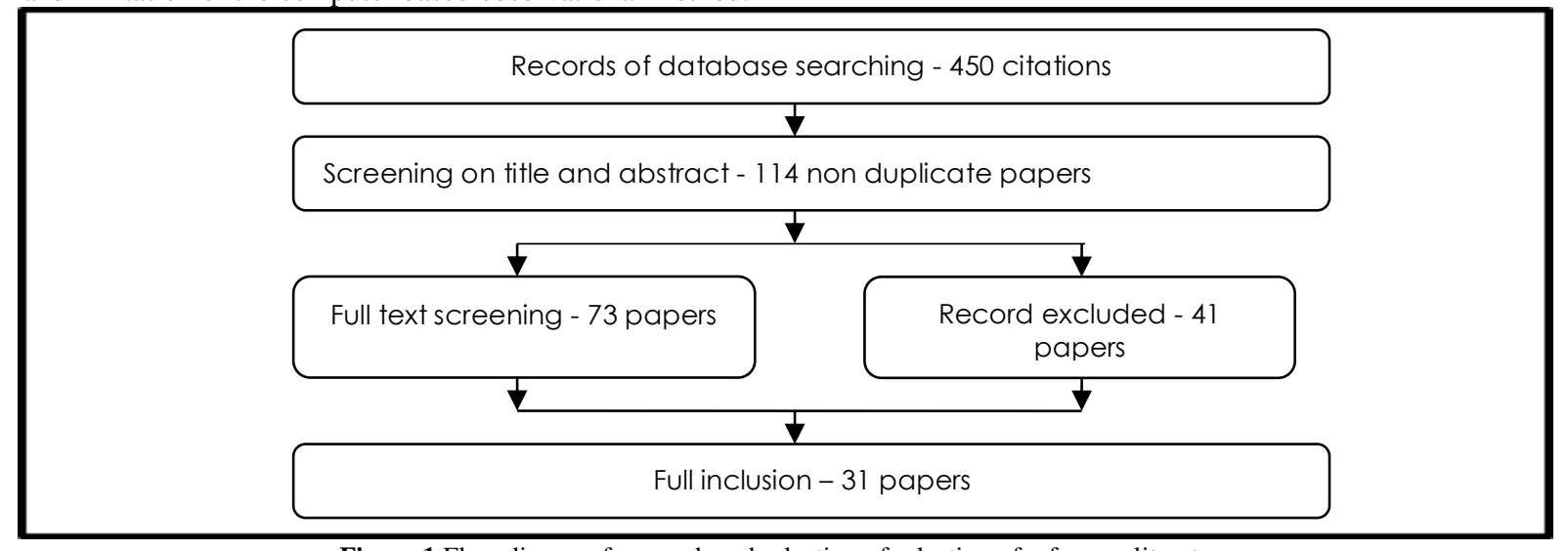

Figure 1 Flow diagram for search and selection of selection of reference literature

\subsection{Evaluation Process}

Selected publications are read by two researchers to evaluate the methods reviewed. The discussions are also needed to get any dissimilarities of information and achieve an agreement to do the documentations of each technique which evaluated based on framework for evaluation in Section 2.2.

\section{Results}

A total of 31 articles are used for final inclusion, that were included seven methods that related to this studies, such as; Ovako Working Posture Analyzing System (OWAS), Ergonomic Analysis (ERGAN), Task Recording and Analysis on Computer (TRAC), Portable Ergonomic Observation (PEO), Hands Relative to The Body (HARBO), Posture, Activity, Tools and Handling (PATH), and Video-and computer-based work analysis (VIDAR).

Table 1 indicates the summary about exposure of risk factors that related to WMSDs. All of the seven tools are covered to assess the exposure of physical risk factors such as; posture and forceful exertion, while only two methods were assessed the psychosocial risk factors. The work organization and individual risk factors are not to be assessed by the existing computer based observational methods. Table 2 shows the evaluation summary of reliability and validity testing. Most of the tools have a good and moderate results.

\subsection{Ovako Working Posture Analysing System (Owas)}

Ovako Working Posture Analysing System (OWAS) is firstly used in the steel industry for assessing physical risk factors including postures for legs and three categories weight of the load handled, where the 252 postures were identified [15, 21, 26]. The method used is based on work sampling with constant time intervals and made as snapshots [21]. The result of interobservers reliability

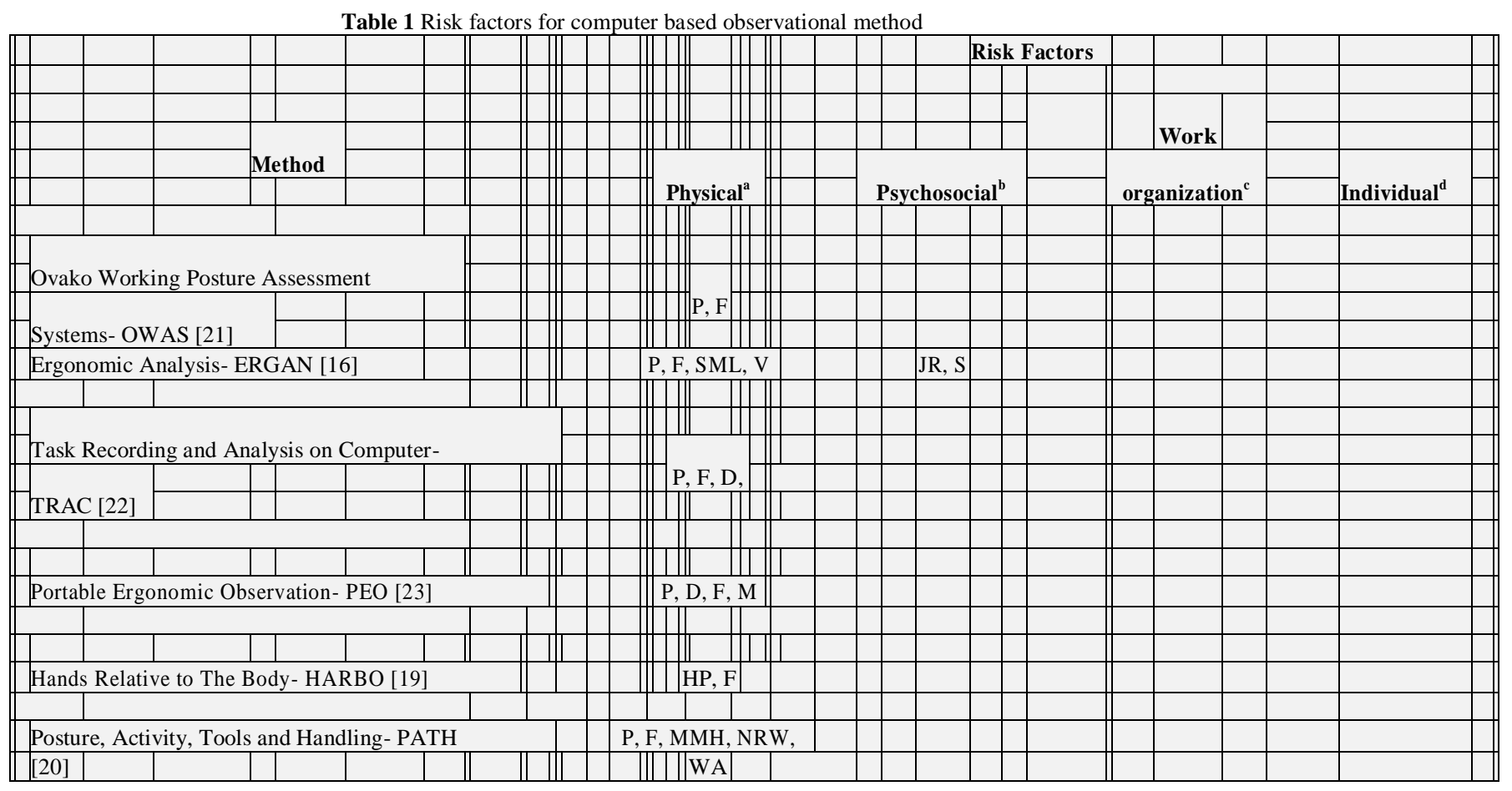




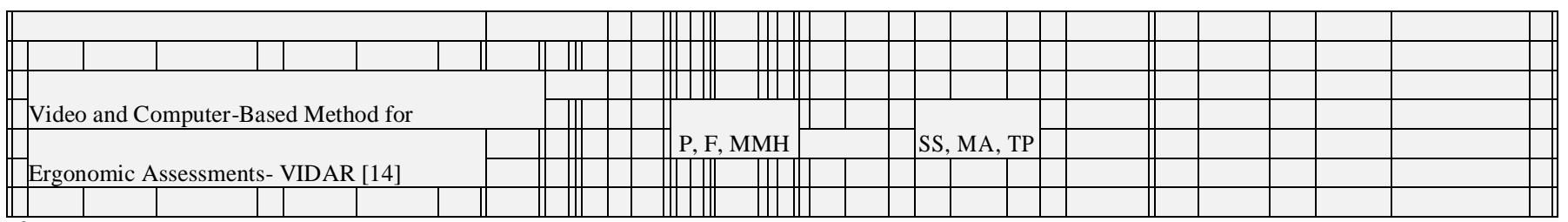

${ }^{\text {a }}$ Physical risk factors including Posture (P), Force (F), Static Muscle Load (SML), Vibration (V), Duration (D), Frequency (F), Movements (M), Hand Posture (HF), Manual Material Handling (MMH), Non-repetitive work (NRW), Work Activity (WA),

${ }^{\mathrm{b}}$ Psychosocial risk factors including Job Rotation (JR), Stress (S), Strain Situation (SS), Mood and Attitude (MA), Time Pressure (TP).

${ }^{\mathrm{c}}$ Work organization factors including work status(fulltime, part-time, temporary), work task(single, rotating), work overload etc.

${ }^{\mathrm{d}}$ Individual factors including anthropometry, age, gender, physical activity, strength etc.

Table 2 Reliability and validity testing of the computer based observational methods

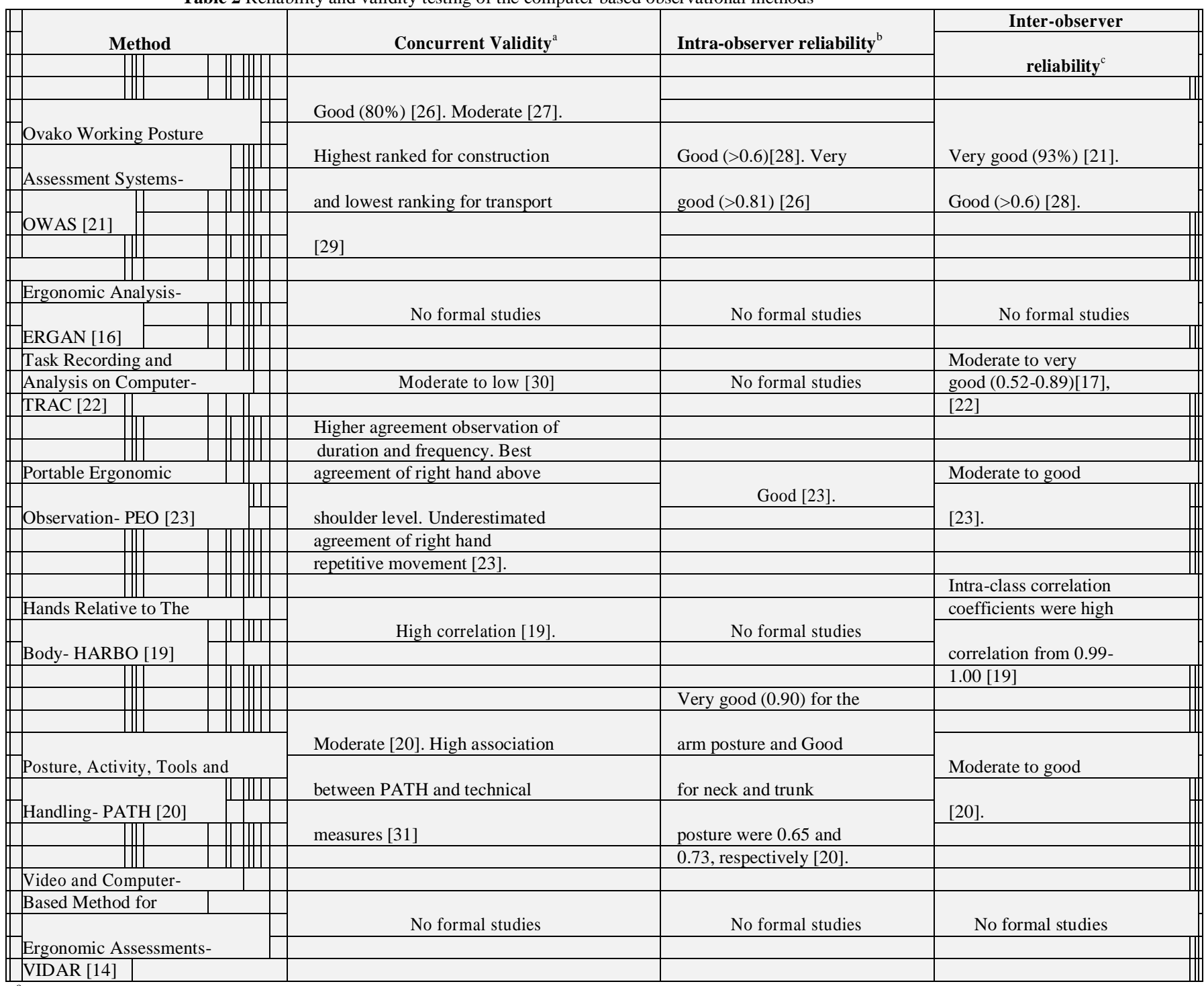

${ }^{\mathrm{a}}$ Concurrent Validity value - The range method correspond with more valid methods: Good, Moderate, Low

${ }^{\mathrm{b}}$ intra-observer reliability value : <0 (less than change agreement), 0.01-0.20 (Poor or Slight agreement), 0.21-0.40 (Fair agreement), 0.41-0.60 (Moderate agreement), 0.61-0.80 (Good or Substantial agreement) and 0.81-0.99 (Very good or Almost perfect agreement) [25]

${ }^{c}$ inter-observer reliability value : $<0$ (less than change agreement), 0.01-0.20 (Poor or Slight agreement), 0.21-0.40 (Fair agreement), 0.41-0.60 (Moderate agreement), 0.61-0.80 (Good or Substantial agreement) and 0.81-0.99 (Very good or Almost perfect agreement)[25] 
testing by Karhu came out with $93 \%$ agreement between the two work-study engineers [21], while Bruijn noted that almost all comparisons between inter-observer and intra-observer reliabilities recorded a percentage over $85 \%$, and the value of kappa is over 0.6 which also considered as a substantial agreement [28]. Consequently, Kayis and Kothiyal found that the result of intraobserver reliability for OWAS, RULA and REBA to be 95\%, 91.7\% and $97.3 \%$, respectively [26]. For concurrent validity, the test obtained moderate result while comparing the OWAS method with direct technical in trunk bending [27], 80\% agreement is found in compare to OWAS, Borg RPE Scale and biomechanical model [26] and higher ranked for construction and lowest ranked for transport [29]. OWAS are easy to used method and can make a faster registration to check a working posture but only focused to assess a back, arm and legs posture.

\subsection{Ergonomic Analysis (ERGAN)}

Ergonomic Analysis (ERGAN) or previously called ARBAN comprises working situation that involves varies body posture, force, static muscle load and vibration for exposure of physical risk factors and also were assesses the psychosocial risk factors including job rotation and work stress [16]. ERGAN are provided a simple and easily handled procedure with a broad area of application. ERGAN is based on sequential studies, working situation that is continuously changing is considered as a series of 'frozen' images at equally long intervals. No formal studies were carried out to test the reliability and validity to construct the tool process.

\subsection{Task Recording and Analysis on Computer (TRAC)}

Task Recording and Analysis on Computer (TRAC) is a method to record the task, actions or posture in real time and time-sampling on a portable computer [17]. The TRAC method only covered to assess the exposure of physical risk factors including posture, force and duration of activities. The inter-observers reliabilities dependent on the body region is between $85 \%$ and $95 \%$ and values of the coefficient kappa is 0.52 and 0.89 , respectively and can be regarded as moderate to very good $[17,22]$. The intra-observer reliabilities were not defined because of the modification between the successive phases, causes reduction of the intra-observer reliability [22]. The result of validity testing is based on dynamic characteristic, where observations against optoelectronic measures in simulated material handling are showed moderate to low correspondence [30]. TRAC is an easy to learn and quick to use methods, but some training are needed to reach the level of performance.

\subsection{Portable Ergonomic Observation (PEO)}

Portable Ergonomic Observation (PEO) is a continuous observation method that is able to investigate posture measurement based on video recording and continuous optoelectronic. Both video recording and continuous optoelectronic were analyzed at the same time during the observation [18]. The objective of this method is to develop a computerized observational method. Based on the observation at the workplace made in real-time where the data were accessed and analyzed immediately. The data collected are based on working postures, duration and frequency during works [23]. Fransson-Hall et al, stated that the outcome of intra-observer and inter-observer reliabilities is good and moderate to good, respectively [23]. Median value and range of minimum and maximum value are used to analyze the validity data and the results are; higher agreement for observation of duration and frequency, best agreement for right hand above shoulder level with the reference value, and underestimated agreement for right hand repetitive movements [23]. PEO also an easy to handle method, but if work pace is quick, only one to three major categories will be observed at one time. These tools was hard to register the left and right hand individually unless the observed work is slow.

\subsection{Hands Relative to the Body (HARBO)}

Hands Relative to the Body (HARBO) method is practical to show an observational technique that is only focusing on positions of the hands in a several hours of recording and the force to be handled [19]. HARBO is applied to register the five standard postures, such as; standing or walking with hand above shoulder level, standing or walking with hands fixed between the shoulder and knuckle level, standing or walking with hand not fixed between the shoulder and knuckle level, standing or walking with hand or hands fixed below the knuckle level, and the stooping, squatting, and kneeling posture and finally is sitting. For each posture, the duration is continuously registered in real time by using handheld computer, and PEO method was used to collect data. For the inter-observer reliability, the twoway analysis of variance is used to compute the intra-class correlation coefficient. Result obtained by the two observers is high for each of the five postures registered and ranged from 0.99 to 1.00 for full day registration. The outcome for the concurrent validity is high correlation compared the HARBO tools with technical measures of the arm and trunk posture [19]. HARBO is a simple and easy to learn method. This method also can be used in various jobs where observers are allowed to assess but time consuming and costly methods.

\subsection{Posture, Activity, Tools and Handling (PATH)}

Posture, Activity, Tools and Handling (PATH) is a computerized work sampling of activity and posture used to analyse non-repetitive work [20]. The PATH data coding is referred to modifications coding system OWAS method and the target exposure for this tool includes the posture, force exertion and manual material handling. The results for intra-observers reliabilities agreement are greater than 0.90 for the leg and arms postures that is classified as excellent agreement. According to the Cohen's kappa analysis for neck and trunk postures, the result is 0.65 and 0.73 , respectively which shows less agreement. However, for inter-observers reliabilities, the result is moderate to good by 0.8 . The result is set as the criterion to initiate data collection of agreement between observers. The outcome of validity trials is moderate association between the PATH trunk posture from video against simulated real time analysis [20], and high association between PATH and technical measures [31] . PATH are suitable to assess for non-repetitive work and have correlation between posture and work activity, but this tools is caused time consuming and several training are required for users to handle the PATH tool.

\subsection{Video-and Computer-Based Work Analysis (VIDAR)}

Video-and computer-based work analysis (VIDAR) or Video-och Datorbaserad Arbetsanalys is an acronym for the Swedish expression which describes the employees' identification towards work tasks that cause pain and discomfort. The method is done by using video display and the outcome will be analysed for ergonomic evaluation [14]. This method also the computer-based program that allows the analysis of physical risk factors including posture and psychosocial condition such as; attitudes and time pressure which are assessed from a video recording of jobs and tasks. By using 
Borg's category ratio (CR-10) scale were assessed by body regions and rates perceived exertion in physical situations. VIDAR is easy to produce report forms and the result are easy to understand. For validity and reliability, no studies testing were found.

\section{Discussions}

The objective of this studies is to help potential users to select the most suitable tool(s) for the specific users and investigate the assessment of risk exposure that causes musculoskeletal disorders, it is the most important stage in the management and prevention of WMSDs in order to ensure the comfort and safety of workers. Most of the existing computer based observational methods have been exposed to physical and psychosocial risk factors that are focusing on postures of the entire body. No general tools can be used to assess the overall types of risk factors.

The concept of validity trials includes some aspects that need to be assessed in terms of concurrent validity. Concurrent validity is the consent of observation in some other methods. Different methods are used simultaneously on the same object that gives different results in several studies. If one of the methods gave more accurate information than the others, it can be considered as a reference for verification. From seven methods that were identified in this study, five have gone through validity testing methods such as OWAS, TRAC, PEO, HARBO and PATH, and they have been evaluated with moderate agreement.

Reliability is measured repeatedly by different people who make measurements on the same thing, at different times, under different conditions. The reliability that was tested in current techniques is intra and inter-observer reliability. Only three tools such as OWAS, PEO and PATH have done the intra-observer reliability, and five tools of computer based observational methods have done the interobserver testing which are; OWAS, TRAC, PEO, HARBO and PATH. The results are mostly good and moderate.

Most computer based observational methods is a simple and easy tool to be learned. They are also more time-saving compared to pen and paper observational methods. Nevertheless, these tools reduce the exposure of risk factors while working.

\section{Conclusion}

There are various computer based observational methods that have been developed to assess exposure to risk factors at workplace. Only some of the methods had its validity and reliability tested. Validity and reliability testing is the most important thing that is needed to consider in developing and validating assessment methods

\section{Acknowledgement}

This research is funded by the Ministry of Higher Education of Malaysia (MOHE) and Universiti Tun Hussein Onn Malaysia (UTHM) under Fundemental Research Grant Scheme (FRGS, Vot 1495)

\section{References}

[1] Barr, A. E. and Barbe, M. F. 2002. Pathophysiological Tissue Changes Associated With Repetitive Movement: A Review of the Evidence. Physical Therapy. 173-187.

[2] Gallagher, S. and Heberger, J. R. 2013. Examining the Interaction of Force and Repetition on Musculoskeletal Disorder Risk a Systematic Literature Review. Human Factors: The Journal of the Human Factors and Ergonomics Society. 55(1): 108-124.
[3] Nunes, I. L. and Bush, P. M. 2012. Work-related Musculoskeletal Disorders Assessment and Prevention. INTECH Open Access Publisher. 1-31.

[4] Widanarko, B., Legg, S., Devereux, J. and Stevenson, M. 2014. The Combined Effect of Physical, Psychosocial/Organisational and/or Environmental Risk Factors on The Presence of Work-Related Musculoskeletal Symptoms and its Consequences. Applied Ergonomics. 45(6): 1610-1621.

[5] Fallentin, N., Juul-Kristensen, B., Mikkelsen, S., Andersen, J. H., Bonde, J. P., Frost, P. and Endahl, L. 2001. Physical Exposure Assessment in Monotonous Repetitive Work-The PRIM Study. Scandinavian Journal of Work, Environment \& Health. 21-29.

[6] Devereux, J. J., Vlachonikolis, I. G. and Buckle, P. W. 2002. Epidemiological Study to Investigate Potential Interaction between Physical and Psychosocial Factors at Work That May Increase the Risk of Symptoms of Musculoskeletal Disorder of the Neck and Upper Limb. Occupational and Environmental Medicine. 59(4): 269277.

[7] Feuerstein, M., Shaw, W. S., Nicholas, R. A. and Huang, G. D. 2004. From Confounders to Suspected Risk Factors: Psychosocial Factors and Work-Related Upper Extremity Disorders. Journal of Electromyography and Kinesiology. 14(1): 171-178.

[8] Sommerich, C. M., Marras, W. S. and Karwowski, W. 2006. WorkRelated Upper Extremity Musculoskeletal Disorders. Handbook of Human Factors and Ergonomics, Third Edition. 855888.Huang, G. D., Feuerstein, M. and Sauter, S. L. 2002. Occupational Stress and Work-Related Upper Extremity Disorders: Concepts and Models. American Journal of Industrial Medicine. 41(5): 298-314

[9] Larsson, B., Søgaard, K. and Rosendal, L. 2007. Work Related NeckShoulder Pain: A Review on Magnitude, Risk Factors, Biochemical Characteristics, Clinical Picture and Preventive Interventions. Best Practice \& Research Clinical

[10] Rheumatology. 21(3): 447-463.

[11] Hooftman, W. E., van Poppel, M. N., van der Beek, A. J., Bongers, P. M. and van Mechelen, W. 2004. Gender Differences in the Relations between Work-Related Physical and Psychosocial Risk Factors and Musculoskeletal Complaints. Scandinavian Journal of Work, Environment \& Health. 30(4): 261-278.

Bodin, J., Ha, C., Serazin, C., Descatha, A., Leclerc, A., Goldberg, M. and Roquelaure, Y. 2012. Effects of Individual and Work-Related Factors on Incidence of Shoulder Pain In A Large Working Population. Journal of Occupational Health. 54(4): 278-288.

[13] David, G. C. 2005. Ergonomic Methods for Assessing Exposure to Risk Factors for Work-Related Musculoskeletal Disorders. Occupational Medicine. 55(3): 190-199.

[14] Kadefors, R. and Forsman, M. 2000. Ergonomic Evaluation of Complex Work: A Participative Approach Employing VideoComputer Interaction, Exemplified In A Study of Order Picking. International Journal of Industrial Ergonomics. 25(4): 435-445. Ahasan, M. R., Väyrynen, S. and Kirvesoja, H. 1996. Physical Workload Analysis among Small Industry Activities Using Postural Data. International Journal of Occupational Safety and Ergonomics. 2(1): 27-34.

Holzmann, P. 1982. ARBAN-A New Method for Analysis of Ergonomic Effort. Applied Ergonomics. 13(2): 82-86.

[17] Frings-Dresen, M. H. and Kuijer, P. P. F. 1995. The TRAC-System: An Observation Method for Analysing Work Demands at the Workplace. Safety Science. 21(2): 163-165.

[18] Leskinen, T., Hall, C., Rauas, S., Ulin, S., Tönnes, M., ViikariJuntura, E. and Takala, E. P. 1997. Validation of Portable Ergonomic Observation (PEO) Method Using Optoelectronic and Video Recordings. Applied Ergonomics. 28(2): 75-83.

Wiktorin, C., Mortimer, M., Ekenvall, L., Kilbom, Å. and Hjelm, E. W. 1995. HARBO, A Simple Computer-Aided Observation Method for Recording Work Postures. Scandinavian Journal of Work, Environment \& Health. 440- 449.

[20] Buchholz, B., Paquet, V., Punnett, L., Lee, D. and Moir, S. 1996. PATH: A Work Sampling-Based Approach to Ergonomic Job Analysis for Construction and Other Non-Repetitive Work. Applied Ergonomics. 27(3): 177-187. 
[21] Karhu, O., Kansi, P. and Kuorinka, I. 1977. Correcting Working Postures in Industry: A Practical Method for Analysis. Applied Ergonomics. 8(4): 199-201.

[22] van der Beek, A. J., van Gaalen, L. C. and Frings-Dresen, M. H. 1992. Working Postures and Activities of Lorry Drivers: A Reliability Study of On-Site Observation and Recording on a Pocket Computer. Applied Ergonomics. 23(5): 331-336.

[23] Fransson-Hall, C., Gloria, R., Kilbom, Å., Winkel, J., Karlqvist, L. and Wiktorin, C. 1995. A Portable Ergonomic Observation Method (PEO) for Computerized On-Line Recording of Postures and Manual Handling. Applied

[24] Ergonomics. 26(2): 93-100.

[25] Vedder, J., 1998. Identifying postural hazards with a video-based occurrence sampling method. International Journal of Industrial Ergonomics, 22(4): 373-380.

[26] Viera, A. J. and Garrett, J. M. 2005. Understanding Inter-Observer Agreement: The Kappa Statistic. Fam Med. 37(5): 360-363.

[27] Kayis, B. and Kothiyal, K. 1996. A Multilevel Approach to Manual Lifting in Manufacturing Industries. International Journal of Occupational Safety and Ergonomics. 2(3): 251-261.

[28] De Bruijn, I., Engels, J. A. and Van der Gulden, J. W. J. 1998. A Simple Method to Evaluate the Reliability of OWAS Observations. Applied Ergonomics. 29(4): 281-283.

[29] Burdorf, A., Derksen, J., Naaktgeboren, B. and van Riel, M. 1992. Measurement of Trunk Bending During Work by Direct Observation and Continuous Measurement. Applied Ergonomics. 23(4): 263-267. van der Beek, A. J., Mathiassen, S. E., Windhorst, J. and Burdorf, A. 2005. An Evaluation of Methods Assessing the Physical Demands of Manual Lifting In Scaffolding. Applied Ergonomics. 36(2): 213-222.

[31] De Looze, M. P., Toussaint, H. M., Ensink, J., Mangnus, C. and Van der Beek, A. J. 1994. The Validity of Visual Observation To Assess Posture In A Laboratory-Simulated, Manual Material Handling Task. Ergonomics. 37(8); 1335-1343.

[32] Paquet, V. L., Punnett, L. and Buchholz, B. 2001. Validity of FixedInterval Observations for Postural Assessment in Construction Work. Applied Ergonomics. 32(3): 215-224. 